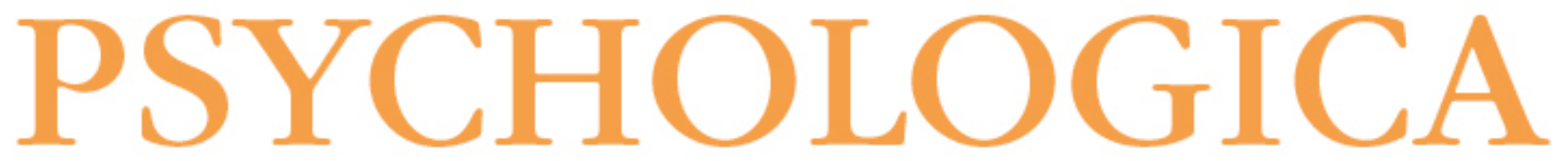

\title{
Caracterização do perfil sócio-demográfico de gestantes adolescentes: revisão da literatura brasileira
}

Autor(es): $\quad \begin{aligned} & \text { Levandowski, Daniela Centenaro; Benetti, Bruna Angélica; Fontoura, } \\ & \text { Laura Ostrowski; Von Hohendorff, Jean; Prati, Laíssa Eschiletti }\end{aligned}$

Publicado por: Imprensa da Universidade de Coimbra

URL

persistente: URI:http://hdl.handle.net/10316.2/5433

DOI: DOI:http://dx.doi.org/10.14195/1647-8606_50_17

Accessed : $\quad$ 26-Apr-2023 11:03:33

A navegação consulta e descarregamento dos títulos inseridos nas Bibliotecas Digitais UC Digitalis, UC Pombalina e UC Impactum, pressupõem a aceitação plena e sem reservas dos Termos e Condições de Uso destas Bibliotecas Digitais, disponíveis em https://digitalis.uc.pt/pt-pt/termos.

Conforme exposto nos referidos Termos e Condições de Uso, o descarregamento de títulos de acesso restrito requer uma licença válida de autorização devendo o utilizador aceder ao(s) documento(s) a partir de um endereço de IP da instituição detentora da supramencionada licença.

Ao utilizador é apenas permitido o descarregamento para uso pessoal, pelo que o emprego do(s) título(s) descarregado(s) para outro fim, designadamente comercial, carece de autorização do respetivo autor ou editor da obra.

Na medida em que todas as obras da UC Digitalis se encontram protegidas pelo Código do Direito de Autor e Direitos Conexos e demais legislação aplicável, toda a cópia, parcial ou total, deste documento, nos casos em que é legalmente admitida, deverá conter ou fazer-se acompanhar por este aviso. 


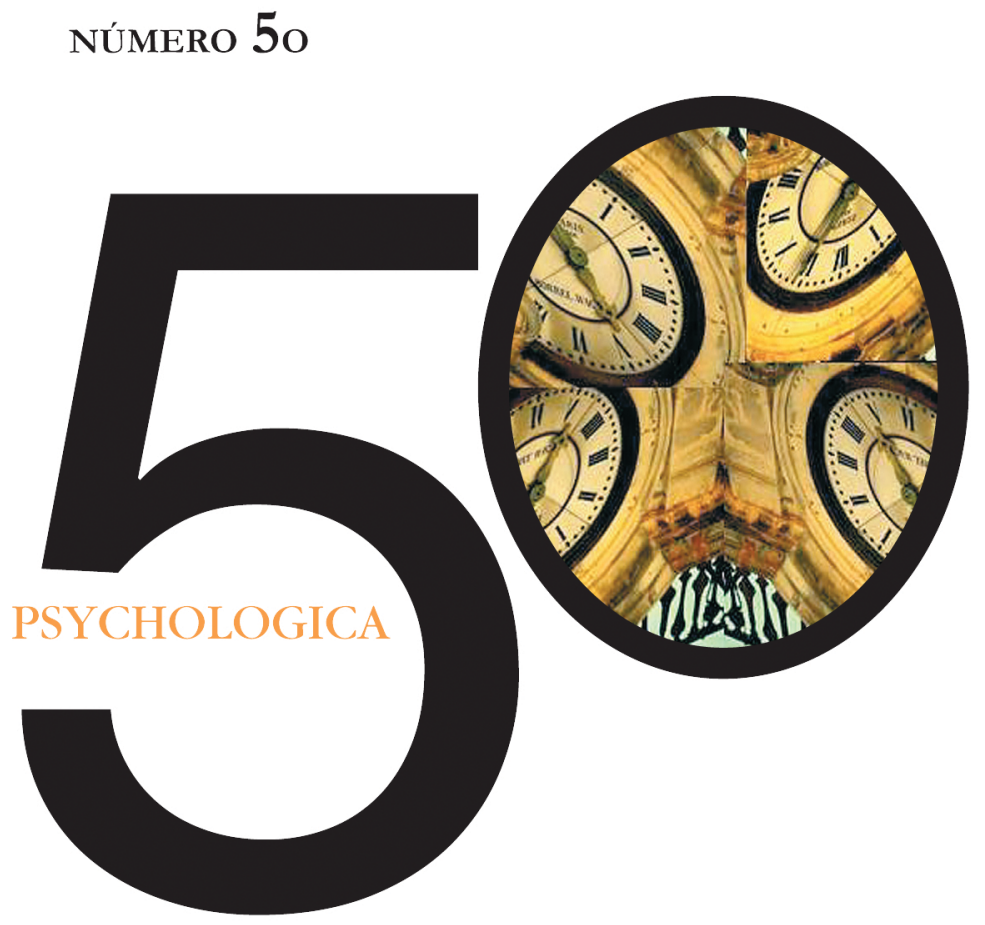

IMPRENSA DA UNIVERSIDADE DE COIMBRA

FACULDADE DE PSICOLOGIA E DE CIÊNCIAS DA EDUCAÇÃO DA UNIVERSIDADE DE COIMBRA 


\section{Caracterização do Perfil Sócio-Demográfico de Gestantes Adolescentes: Revisão da Literatura Brasileira'}

Daniela Centenaro Levandowski², Bruna Angélica Benetti ${ }^{3}$, Laura Ostrowski Fontoura4, Jean Von Hohendorffs \& Laíssa Eschiletti Prati ${ }^{6}$

O presente estudo apresenta uma pesquisa bibliográfica de estudos brasileiros publicados e/ou produzidos entre 1987 e 2007, que investigaram o perfil sócio-demográfico de gestantes adolescentes atendidas em instituições públicas de saúde. A partir da realização de uma pesquisa em sites de busca (Scielo, BVS-Psi, Google Acadêmico), foram localizados 23 trabalhos sobre o tema, que foram analisados em relação às características informadas. Verificou-se que em geral a faixa etária considerada para análise foi de 10 a 19 anos, sendo o número de gestações mais elevado em jovens de nível socioeconômico baixo e com 17 anos. Também se evidenciou carência de informações que proporcionassem a caracterização de aspectos relacionais e familiares, o que pode dificultar o planejamento de intervenções junto a essa população. Sugere-se que estudos futuros incluam outras informações relevantes para a caracterização de um perfil sócio-demográfico ampliado dessa clientela, como dados do parceiro e dos genitores da adolescente.

PALAVRAS-CHAVE: Perfil Sócio-demográfico; Gravidez na Adolescência; Pesquisa Bibliográfica.

\footnotetext{
1 O presente trabalho deriva do Projeto de Pesquisa "Caracterização da maternidade na adolescência no Vale Do Paranhana: Perfil sócio-demográfico, expectativas e vivências das jovens, seus familiares e parceiros", desenvolvido pela Profa. Dra. Daniela Centenaro Levandowski no ano de 2007, nas Faculdades de Taquara (FACCAT), e financiado por essa mesma instituição.

2 Psicóloga, Mestre em Psicologia e Doutora em Psicologia do Desenvolvimento (UFRGS), Docente do Programa de Pós-Graduação em Psicologia da Universidade do Vale do Rio dos Sinos. Docente do Curso de Psicologia das Faculdades de Taquara (FACCAT) de Fevereiro de 2004 a Janeiro de 2008.

3 Acadêmica do Curso de Psicologia da FACCAT, Bolsista de Iniciação Científica da FACCAT no referido projeto de pesquisa.

4 Acadêmica do Curso de Psicologia da FACCAT e auxiliar voluntária de pesquisa no referido projeto. 5 Acadêmico do Curso de Psicologia da FACCAT e auxiliar voluntário de pesquisa no referido projeto. 6 Psicóloga, Doutoranda em Psicologia (UFRGS) e Docente do Curso de Psicologia das Faculdades Integradas de Taquara (FACCAT).
} 


\section{Introdução}

A adolescência é uma fase de mudanças, de readaptação ao novo corpo e de novas atitudes frente à vida (Godinho, Schelp, Parada, \& Bertoncello, 2000). De fato, nessa etapa o adolescente vive a perda do corpo e da identidade infantil e, de um momento outro, se depara com a necessidade de redefinir sua imagem corporal e sua identidade (Aberastury \& Knobel, 1970/1981). Logo, a adolescência caracteriza-se por ser um período de profunda instabilidade emocional (Silveira, Oliveira, \& Fernandes, 2004).

Adicionando-se a esse contexto uma gravidez, compreender-se-á como esse acontecimento pode ser difícil para a adolescente (Godinho et al., 2000). Nesse momento do ciclo vital individual, a gravidez pode ter sérias implicações biológicas, familiares, psicológicas e sociais. Por exemplo, essa condição pode limitar ou adiar as possibilidades de engajamento social dessas jovens, especialmente do ponto de vista econômico (Carniel, Zanoli, Almeida \& Morcillo, 2006; Ponte Junior \& Ximenes Neto, 2004). Além disso, pode promover a evasão escolar, dependendo das características do contexto em que a jovem está inserida (Chalem, Mitsuhiro, Ferri, Barros, Guinsburg \& Laranjeira, 2007). Em relação às implicações biológicas, verifica-se que a imaturidade física da mãe associa-se a maiores taxas de baixo peso, prematuridade e mortalidade do bebê (Simões, Silva, Bettiol, Lamy-Filho, Tonial, \& Mochel, 2003).

Pelo exposto, fica evidente que as adolescentes grávidas necessitam de um apoio efetivo, incluindo-se aí uma assistência pré-natal integral e especializada, de caráter multidisciplinar. As intervenções devem facilitar o exercício de seu papel materno, em função das inúmeras demandas com as quais se deparam simultaneamente (pessoais, conjugais, familiares e sociais) (Levandowski, 2005). Neste sentido, o apoio dado às adolescentes é primordial para que essas tolerem as mudanças biopsicossociais decorrentes da vivência simultânea da adolescência e da maternidade (Piccinini, Rapoport, Levandowski, \& Voigt, 2002).

Com o objetivo de melhor atender esse público, surge a necessidade de se investigar de forma mais aprofundada o perfil sócio-demográfico dessas jovens. Esse conhecimento permitirá o desenvolvimento de intervenções e políticas públicas mais adequadamente direcionadas para as necessidades dessa parcela da população, constituindo-se em uma fonte importante de apoio. Além disso, não se pode esquecer que, no Brasil, são freqüentes os relatos do aumento do índice de gestações nesta faixa etária (Carniel et al., 2006; Sabroza, Leal, Gama, \& Costa, 2004), a ponto de essa situação já ser considerada um problema de saúde pública (Chalem et al., 2007). 


\section{Método}

O procedimento metodológico abrangeu um levantamento, através de consulta a sites especializados (Scielo, BVS-Psi e Google Acadêmico), de artigos empíricos, monografias de especialização, dissertações e teses elaboradas no contexto brasileiro, que focalizaram a caracterização do perfil sócio-demográfico de gestantes adolescentes atendidas em instituições de saúde. Foram considerados estudos sobre o tema produzidos e/ou publicados entre 1987 e 2007.

Para a realização da busca, foram utilizados os seguintes termos descritores, combinados alternadamente: gravidez, adolescência, perfil, sócio-demográfico. A partir daí, foram localizados aproximadamente 50 trabalhos. Muitos não se encontravam disponíveis, tendo sido automaticamente eliminados. Seguindo o objetivo de caracterização de perfil sócio-demográfico de gestantes adolescentes, 30 trabalhos foram selecionados. Após uma primeira leitura, foram descartados sete estudos por apresentarem informações bastante incompletas, o que inviabilizava a caracterização de tal perfil. Sendo assim, foram analisados efetivamente 23 estudos, assim distribuídos: 19 artigos científicos, três Dissertações de Mestrado e uma Monografia de Curso de Especialização.

Procedeu-se, então, a uma nova leitura dos 23 trabalhos selecionados, destacando-se os principais aspectos informados por esses estudos. De posse de tal panorama, foram selecionados os aspectos que mais se destacavam na caracterização do perfil das gestantes adolescentes, por serem informados na maioria dos estudos. Os seguintes aspectos foram localizados nos trabalhos: condição e faixa etária, nível socioeconômico, condição escolar, ocupação, número de gestações, idade da menarca e da primeira relação sexual, conhecimento sobre métodos contraceptivos e uso dos mesmos, planejamento da gravidez e tentativa de aborto, uso de álcool e/ou cigarro e drogas ilícitas, abuso físico e/ou sexual e momento de ocorrência, realização de acompanhamento pré-natal e estado civil. Após isso, essas informações foram organizadas, visando a facilitar o seu entendimento e análise. São apresentados, a seguir, o delineamento dos estudos explorados, os aspectos recém mencionados e, na seqüência, uma discussão sobre as principais informações coletadas.

\section{Resultados e Discussão}

\subsection{Delineamento dos estudos}

Em função do tipo de temática investigada, destacou-se entre os estudos localizados a abordagem quantitativa e descritiva. Grande parte desses estudos utilizou uma abordagem comparativa entre diferentes grupos de gestantes adolescentes, 
divididas em função da idade (Sabroza, 2002; Simões et al., 2003), ou entre gestantes adolescentes e adultas jovens (Alegria, Schor, \& Siqueira, 1989; Costa et al., 1999; Gama, Szwarcwald, \& Leal, 2002; Madi, Bertotto, \& Ribeiro, 2001; Pinto, Malafaia, Borges, Baccaro, \& Soranz, 2005).

326 Alguns estudos também tiveram um caráter transversal (Carniel et al., 2006; Chalem et al., 2007). Apenas dois estudos caracterizaram-se como longitudinais, investigando a gestação e as modificações no periodo perinatal (Madi et al., 2001; Simões et al., 2003).

Dentre os estudos revisados, vários foram realizados a partir de consulta aos prontuários ou outros registros das gestantes, como Declaração de Nascidos Vivos, caracterizando-se como pesquisas documentais (Beretta, Denari, \& Pedrazzani, 1995; Carniel et al., 2006; Rigol \& Santo, 2001; Silveira, Oliveira \& Fernandes, 2004; Zaganelli, 2006; Zanchi, Carneiro, \& Menezes, 1999).

\subsection{Dados das amostras dos estudos}

\subsubsection{Condição e faixa etária}

Quanto à condição da amostra (gestante x puérpera), pode-se verificar que 11 estudos avaliaram as adolescentes durante a gestação (Alegria et al., 1989; Chalem et al., 2007; Chemelo, Tanaka, Buzzetti, \& De Lorenzi, 2001; Madi et al., 2001; Persona, Shimo, \& Tarallo, 2004; Pinto et al., 2005; Ponte Júnior \& Ximenes Neto, 2004; Rigol \& Santo, 2001; Velasco, 1998; Viana, 2005; Zanchi et al., 1999). Dentre esses, um artigo ponderou a repetição da gestação (Persona et al., 2004). Também onze estudos contemplaram a situação das adolescentes no puerpério (Aquino et al., 2003; Beretta et al., 1995; Carniel et al., 2006; Costa et al., 1999; Faloppa et al., 1994; Gama et al., 2002; Goldenberg et al., 2005; Sabroza, 2002; Silveira et al., 2004; Simões et al., 2003; Zaganelli, 2006). Apenas um estudo investigou o perfil de gestantes e puérperas concomitantemente (Lima, Kobata, \& Silvestrini, 2000).

A variação da faixa etária mais considerada para análise foi de 10 a 19 anos, delimitação análoga à utilizada pela Organização Mundial de Saúde (OMS; www. who.int). Apenas o artigo de Aquino et al. (2003) considerou adolescentes entre 18 e 24 anos, variação de idade bastante discrepante dos demais estudos, mas adequada ao objetivo dos autores, que foi conhecer os desdobramentos posteriores de uma gravidez na adolescência.

Em relação ao tamanho da amostra, percebe-se que o número de participantes nos estudos variou de 18 a 2563 adolescentes gestantes/puérperas. Essa variação expressiva no tamanho da amostra está diretamente relacionada à metodologia e aos objetivos de cada trabalho. Além disso, pode-se pensar que tal variação 
seja também decorrente do número de pesquisadores envolvidos, do tempo disponível para a realização da pesquisa e das parcerias e incentivos obtidos, aspectos que podem facilitar uma abrangência maior de casos. Também não se pode desconsiderar que somente o acesso às participantes pode ter contribuído para essas diferenças no tamanho das amostras, uma vez que alguns estudos se basearam na consulta a documentos, enquanto outros coletaram os dados junto às adolescentes.

\subsubsection{Nível sócio-econômico}

Dos 23 estudos revisados, apenas oito (Aquino et al., 2003; Chalem et al., 2007; Chemello et al., 2001; Madi et al., 2001; Persona et al., 2004; Pinto et al., 2005; Ponte Junior \& Ximenes Neto, 2004; Simões et al., 2003) referiram a renda ou nível sócio-econômico da amostra analisada. É possível constatar um número mais elevado de gestações entre adolescentes de camadas sociais mais baixas, o que corresponde ao nível sócio-econômico baixo. Por exemplo, de acordo com o estudo de Chemello et al. (2001), a renda de 69,7\% da amostra era de até 0,25 salários mínimos brasileiros.

Alguns estudos citaram aspectos específicos que permitiram obter um melhor esclarecimento sobre a situação sócio-econômica dessas gestantes, tais como local de residência (favela/rua; Gama et al., 2002; Sabroza, 2002) e falta de água canalizada e rede de esgoto (Pinto et al., 2005).

Pode-se inferir, contudo, que a maior incidência de gravidez na adolescência na população de baixa renda, constatada nesses estudos, seja um viés derivado dos locais utilizados para a coleta de dados, em geral hospitais ou outros órgãos públicos de saúde. As gestantes de nível sócio-econômico mais elevado na maioria das vezes não utilizam tais serviços. Os dados referentes a atendimentos para essa parcela da população são escassos (nesta investigação, não foram encontradas pesquisas com essa clientela).

\subsubsection{Condição escolar}

Com base nos nove estudos que informaram a condição escolar das participantes, verificou-se uma maior incidência de adolescentes gestantes ou puérperas que referiram não estar estudando no momento da investigação (Aquino et al., 2003; Beretta et al., 1995; Chalem et al., 2007; Chemello et al., 2001; Faloppa et al., 1994; Persona et al., 2004; Ponte Junior \& Ximenes Neto, 2004; Rigol \& Santo, 2001). Algumas adolescentes indicaram a interrupção dos estudos antes da ocorrência da gravidez, e não apenas como conseqüência desse estado (Sabroza, 2002). Os estudos também salientaram o não retorno à escola após o nascimento do bebê (Aquino et al., 2003; Faloppa et al., 1994). 
Apenas dois trabalhos (Faloppa et al., 1994; Ponte Junior \& Ximenes Neto, 2004) referiram os motivos alegados pelas participantes para o abandono dos estudos: devido à gravidez, por necessidade de trabalhar, por opção e por falta de escola próxima ao local de moradia. Pensa-se que o fato de estar grávida por si só parece ocasionar uma possibilidade de abandono da escola, seja pela vergonha ou pela necessidade de trabalhar para auxiliar no sustento do bebê. Pode-se pensar ainda que o fato de gerar uma criança faça com que a adolescente sinta-se adulta, não considerando mais a escola como um local importante e necessário frente às suas novas demandas. Todavia, não se pode esquecer que aquelas adolescentes que possuem parceiros podem escolher dedicar-se ao lar e à criança. Parece, entretanto, ficar claro em todas essas situações que a escolarização não assume uma posição de destaque na vida da adolescente, talvez nem mesmo antes da gravidez, gerando, assim, a expectativa de outras possibilidades de inserção social, por exemplo, via maternidade.

\subsubsection{Ocupação}

Embora nove estudos revisados não tenham acessado essa informação, aqueles trabalhos que investigaram tal aspecto (Aquino et al., 2003; Beretta et al., 1995; Carniel et al., 2006; Chalem et al., 2007; Chemello et al., 2001; Costa et al., 1999; Faloppa et al., 1994; Gama et al., 2002; Lima et al., 2000; Persona et al., 2004; Pinto et al., 2005; Ponte Junior \& Ximenes Neto, 2004; Rigol \& Santo, 2001; Sabroza, 2002; Simões et al., 2003) indicaram que o número de gestantes que não apresentavam uma ocupação definida foi expressivamente superior ao daquelas que relataram ter uma ocupação. Interessante observar que, em alguns estudos, as adolescentes mencionaram não ter projetos profissionais (Lima et al., 2000) ou não pretender trabalhar futuramente (Sabroza, 2002).

Dentre as ocupações das adolescentes, pode-se verificar que as mais citadas foram do lar, estudante, empregada doméstica e serviços gerais. Isso indica atividades de baixa remuneração, cujo nível de escolaridade exigido para o seu desempenho também é baixo. Tal fato pode ser explicado parcialmente pelos dados mencionados anteriormente. Uma vez que a adolescente grávida (ou até mesmo antes disso) abandona os estudos, não consegue adquirir uma formação educacional suficiente para facilitar o seu acesso ao mercado de trabalho. Sendo assim, a umenta a probabilidade de ficar desempregada ou de exercer funções que não demandem conhecimentos específicos. Ao mesmo tempo, não tendo estudo e capacitação profissional, a adolescente parece excluir de sua trajetória projetos profissionais, uma vez que, sem o preparo necessário, as possibilidades de êxito e crescimento profissional tornam-se ainda mais escassas. 


\subsubsection{Número de gestações}

Dezesseis trabalhos revisados investigaram o número de gestações das participantes (Beretta et al., 1995; Carniel et al., 2006; Chalem et al., 2007; Chemello et al., 2001; Costa, et al., 1999; Lima et al., 2000; Madi et al., 2001; Persona et al., 2004; Pinto et al., 2005; Ponte Junior \& Ximenes Neto, 2004; Rigol \& Santo, 2001; Simões et al. 2003; Velasco, 1998; Viana, 2005; Zaganelli, 2006; Zanchi et al., 1999). Em todos os trabalhos, a incidência de gestantes primigestas foi superior, variando de $62 \%$ (Simões et al., 2003) a 91\% (Chalem et al., 2007). Tal dado pode estar relacionado à idade das participantes, pois as mais jovens, levando em consideração a proximidade da idade da menarca e da idade da gestação, não haveriam tido tempo suficiente para engravidar mais de uma vez. Por outro lado, se pensarmos que a grande maioria das adolescentes pesquisadas engravidou por volta dos 17 anos, essa informação indicaria uma maior preocupação com a ocorrência da gestação, levando ao uso de métodos contraceptivos já no início da vida sexual.

A incidência de gestantes secundigestas apresentou uma expressiva variação entre os estudos, de 6\% (Ponte Junior \& Ximenes Neto, 2004) a 88,89\% (Persona et al., 2004). Diante dessa discrepância, inferimos que talvez a diferença de faixa etária das adolescentes pesquisadas seja responsável por esse panorama, pois aquelas adolescentes que tiveram o primeiro filho precocemente apresentam uma maior probabilidade de terem sido mães pela segunda vez ainda na adolescência, dentro da faixa de idade considerada na maioria dos trabalhos analisados.

A maioria dos estudos informou até a terceira e quarta gestação das participantes (Beretta et al., 1995; Madi et al., 2001; Persona et al., 2004; Pinto et al., 2005; Ponte Junior \& Ximenes Neto, 2004; Velasco, 1998; Zanchi et al., 1999). Apenas dois estudos mencionaram mais de quatro gestações (Lima et al., 2000; Simões et al., 2003) e destes, um chegou a informar a sexta gestação da adolescente (Lima et al., 2000). É preocupante tal recorrência de gestações, pois faz crer que essas adolescentes iniciaram precocemente sua vida sexual e reprodutiva, provavelmente por falta de informações e/ou uso incorreto (ou não uso) de medidas contraceptivas e que, além disso, após a ocorrência da primeira gestação, não passaram a prevenir-se para evitar uma nova gravidez. Os dados também fazem pensar que o intervalo de tempo entre cada gestação não foi muito longo, o que reforça a idéia de uma falta de aprendizagem e de reflexão dessas adolescentes sobre a sua condição e as conseqüências dela a curto, médio e longo prazo.

Alguns estudos não especificaram o número preciso de gestações das participantes, usando uma classificação mais geral, como "duas gestações ou mais" ou "não era a primeira gestação" (Carniel et al., 2006; Chalem et al., 2007; Costa et al., 1999; Rigol \& Santo, 2001; Simões et al., 2003; Velasco, 1998; Viana, 2005). O fato de os estudos não especificarem tal número dificulta a obtenção de uma informação 
mais objetiva a esse respeito e, conseqüentemente, o estabelecimento de um perfil. Por se tratar de um dado facilmente identificável junto às gestantes, sugere-se que seja melhor explicitado em estudos futuros.

\subsubsection{Idade da menarca e da primeira relação sexual}

Apenas seis trabalhos abordaram a idade da menarca das participantes (Chemello et al.,2001; Costa et al., 1999; Lima et al., 2000; Persona et al., 2004; Pinto et al., 2005; Ponte Junior \& Ximenes Neto, 2004), apesar da importância dessa informação para a elaboração de programas de prevenção à gravidez. A partir desses estudos, percebeu-se que essa variou de oito a 16 anos, sendo a média de idade mais citada de 12 a 13 anos.

Em relação à idade de início da atividade sexual, pode-se averiguar que oito trabalhos referenciaram o tema (Aquino et al., 2003; Chalem et al., 2007; Chemello et al., 2001; Costa et al., 1999; Faloppa et al., 1994; Persona et al., 2004; Pinto et al., 2005; Ponte Junior \& Ximenes Neto, 2004). Novamente destaca-se a falta de uma informação essencial para a caracterização do perfil das adolescentes grávidas, pois permite estimar o tempo que a adolescente leva entre o início da atividade sexual e a ocorrência da gravidez, dado imprescindivel para ser considerado na elaboração de intervenções visando à prevenção dessa situação. De acordo com os referidos estudos, tal idade variou de nove a 19 anos. No entanto, a maioria das adolescentes iniciou sua vida sexual entre 14 e 19 anos de idade.

Através destes dados, pode-se deduzir que a idade de início da atividade sexual pode ser, em alguns casos, anterior à menarca (nove anos), o que indica a atual precocidade da iniciação sexual dos adolescentes brasileiros. A iniciação sexual após a menarca não é menos preocupante, pois aumenta a possibilidade de uma gravidez, ainda mais se levando em consideração o fato de as adolescentes não se preocuparem com a utilização de métodos anticonceptivos, como ficou evidenciado pelos altos índices de recorrência da gestação.

Embora conhecer a idade de início da atividade sexual seja importante, não se pode esquecer de investigar o uso e o conhecimento de métodos contraceptivos dos adolescentes, uma vez que são tais métodos que podem diminuir a prevalência de gravidez nessa faixa etária.

\subsubsection{Conhecimento sobre métodos contraceptivos e uso dos mesmos}

Dos estudos revisados, seis deles abordaram o conhecimento das jovens acerca dos métodos contraceptivos (Aquino et al., 2003; Chemello et al., 2001; Costa et al., 1999; Faloppa et al., 1994; Pinto et al., 2005; Ponte Junior \& Ximenes Neto, 2004) e sete caracterizaram o uso de tais métodos (Aquino et al., 2003; Chalem 
et al., 2007; Costa et al., 1999; Faloppa et al., 1994; Persona et al., 2004; Pinto et al., 2005; Sabroza; 2002).

O não uso de método contraceptivo entre os estudos variou de 81,8\% (Sabroza, 2002) a 11,1\% (Persona et al., 2004). Alguns estudos (Faloppa et al., 1994; Ponte Junior \& Ximenes Neto, 2004) buscaram detalhar tal uso entre as adolescentes. A investigação de Chalem et al. (2007) evidenciou que 43,2\% das participantes nunca haviam utilizado preservativo durante as relações sexuais, enquanto $21,5 \%$ referiram sempre haver utilizado. Este mesmo estudo investigou o uso de método anticoncepcional na época em que a gravidez ocorreu. Dentre as participantes, $76,2 \%$ não estavam fazendo uso de nenhum método e $23,8 \%$ faziam uso, provavelmente incorreto, de contraceptivos, o que acarretou a gestação. Tais dados nos fazem refletir acerca da importância e da necessidade de informar os jovens para conscientizá-los acerca do uso de métodos contraceptivos, não apenas para prevenir a ocorrência de uma gravidez não planejada, como também a contaminação por alguma doença sexualmente transmíssivel.

Quanto aos métodos utilizados, o estudo de Persona et al. (2004) demonstrou a predominância da pílula anticoncepcional (55,55\%), seguida de preservativo masculino (27,78\%) e hormônio injetável (22,23\%). O estudo de Faloppa et al. (1994) buscou identificar o conhecimento das participantes sobre os diferentes métodos contraceptivos: $86 \%$ referiram conhecer a pílula anticoncepcional, $78 \%$ o preservativo masculino, $36 \%$ a tabelinha, $24 \%$ o DIU (Dispositivo Intra-Uterino) e $16 \%$ o coito interrompido. Contudo, chamou mais atenção o desconhecimento das jovens acerca desses métodos (14\%, 22\%, 64\%, $76 \%$ e $84 \%$, respectivamente). Percebe-se a existência de métodos contraceptivos mais conhecidos pela população adolescente brasileira (como o preservativo masculino e a pílula) em comparação a outros (tabelinha, DIU e coito interrompido), talvez até mesmo porque as campanhas de prevenção realizadas focam tais métodos. Diante disso, pensa-se que tais campanhas estejam surtindo algum efeito entre essa população, uma vez que são referidos como os métodos mais conhecidos e utilizados. Além disso, tais métodos são de fácil acesso por parte da população, uma vez que muitos postos de saúde os distribuem gratuitamente.

De qualquer modo, é notável que atualmente a maioria das gestantes, mesmo conhecendo algum método contraceptivo, não os utilize e se arrisque em relações sexuais desprotegidas, o que explica a alta incidência de gestações e de repetições de gestações identificadas anteriormente. Portanto, o conhecimento dos métodos contraceptivos não parece ser suficiente para garantir o seu uso.

Pode-se verificar que apenas Aquino et al. (2003) buscaram identificar a fonte de informação da adolescente sobre aspectos da sexualidade: a mãe $(23,6 \%)$, o pai $(12,4 \%)$ e a escola $(19,4 \%)$. Diante disso, não se pode deixar de comentar a 
importância que a escola e a família têm para a adolescente, especialmente no que se refere à educação sexual. Tal informação indica a necessidade de se investir na formação de educadores sexuais em nosso país e de se intervir junto aos pais de adolescentes, para que se sintam seguros para educar os filhos em relação a esse aspecto de suas vidas. Isso porque a situação atual parece remeter à falta de uma educação sexual entre os jovens, que deveria ser construída conjuntamente pela família e pela escola. Não se pode desconsiderar, todavia, a influência de aspectos individuais dos próprios adolescentes, tais como a imaturidade cognitiva (no caso, a sensação de invulnerabilidade e a onipotência; Papalia, Olds \& Feldman, 2001/2006), que os impelem ao não uso de medidas contraceptivas.

\subsubsection{Planejamento da gravidez e tentativa de aborto}

Nove trabalhos abordaram o planejamento da gravidez pelas participantes, referido algumas vezes como o desejo de ser mãe (Aquino et al., 2003; Chalem et al., 2007; Chemello et al., 2001; Gama et al., 2002; Madi et al., 2001; Persona et al., 2004; Pinto et al., 2005; Ponte Junior \& Ximenes Neto, 2004; Sabroza, 2002). Pode averiguar-se, a partir disso, a maior incidência de não planejamento da gravidez entre as adolescentes, que variou de $50 \%$ a $81,2 \%$.

O aborto foi referido pelas participantes em sete trabalhos (Aquino et al., 2003; Gama et al., 2002; Pinto et al., 2005; Ponte Junior \& Ximenes Neto, 2004; Sabroza, 2002; Viana, 2005; Zanchi et al., 1999). Destes, seis referiam histórico de aborto ou tentativa de aborto. No estudo conduzido por Viana (2005), 14\% das participantes haviam realizado aborto. $O$ estudo de Aquino et al. (2003) detalhou os tipos de aborto ocorridos: 9,6\% das jovens relataram aborto espontâneo e 15,3\%, aborto provocado. No estudo de Sabroza (2002), 13\% das participantes de 12 a 16 anos alegaram tentativa de aborto, mas esse índice foi ainda maior entre as adolescentes de 17 a 19 anos (27,2\%). Além disso, Ponte Junior e Ximenes Neto (2004) evidenciaram que $12 \%$ das participantes de seu estudo pensaram em realizar aborto e só não o efetivaram por falta de condições financeiras.

Esses dados indicam que o aborto surge como uma solução para a gravidez indesejada. Assim, quando planejado ou desejado, serve para o casal (ou somente a adolescente) "resolver" a situação, evidenciando um modo de solucionar a "dificuldade" bastante arriscado física e emocionalmente. No Brasil, essa prática não é legalizada e nem sempre os adolescentes têm consciência das repercussões emocionais dessa decisão. A falta de estrutura emocional, financeira e social pode também colaborar para a decisão por tal medida. Assim, não se pode esquecer que uma gravidez não planejada pode desestruturar emocionalmente a jovem, levando-a a apresentar comportamentos-limite. Nos casos dos abortos espontâneos, pensa-se que a precocidade da gestação, a falta de acompanhamento pré-natal 
e as situações de vida e saúde (alimentação, saneamento básico, moradia, etc.) podem influenciar para a sua ocorrência.

\subsubsection{Uso de álcool e/ou cigarro e drogas ilícitas}

No que concerne ao consumo de álcool e/ou cigarro durante a gestação, apenas seis estudos abordaram o tema (Chalem et al., 2007; Gama et al., 2002; Pinto et al., 2005; Sabroza, 2002; Simões et al., 2003; Viana, 2005), apesar de sua importância para a saúde da gestante e do feto. Os índices de consumo de álcool variaram de $6,9 \%$ a $26,6 \%$ entre os estudos, enquanto que os de cigarro, de $3,1 \%$ a $27,3 \%$. Por fim, o uso de drogas ilícitas variou de $0,6 \%$ a 1,7\% nas amostras estudadas. Apenas o estudo de Chalem et al. (2007) referiu os tipos de drogas ilícitas consumidas pelas participantes: maconha, cocaína e droga injetáveis.

Pode-se verificar que o uso de álcool e/ou cigarro foi superior ao consumo de drogas ilícitas na gestação. Tais drogas são de mais fácil acesso, não são caras e socialmente não são vistas como drogas propriamente ditas (são lícitas). Contudo, embora nem todas as gestantes utilizem drogas ilícitas, isso não significa necessariamente menor prejuízo para o desenvolvimento do feto e maior consciência frente à maternidade.

\subsubsection{Ocorrência de abuso físico e/ou sexual}

Cinco artigos abordaram a ocorrência de abuso físico ou sexual da adolescente (Chalem et al., 2007; Gama et al., 2002; Madi et al., 2001; Ponte Junior \& Ximenes Neto, 2004; Sabroza, 2002) e dentre esses, apenas um estudo não relatou o momento da ocorrência do abuso (Ponte Junior \& Ximenes Neto, 2004). Com base nisso, pode-se verificar que a incidência de abuso físico ou sexual variou de $4 \%$ a $27 \%$. Os tipos de abuso relatados foram: ataques com arma de fogo, violência sexual, vivência de agressão física, ameaça de violência em geral e violência física. No estudo de Ponte Junior e Ximenes Neto (2004) constavam informações sobre o agressor: em geral o pai, seguido pelo parceiro e pela mãe. Em relação ao momento da ocorrência da violência física ou sexual, dois estudos relataram ter ocorrido durante a gestação (Gama et al., 2002; Sabroza, 2002), um estudo referiu durante a adolescência (Madi et al., 2001) e um outro, antes e durante a gestação (Chalem et al., 2007).

Certamente que não podem ser pensadas justificativas plausiveis para tal realidade, mas a submissão da vítima, por medo de maior punição em função de denúncia, pode contribuir para perpetuar essa situação. A gravidez indesejada pelo parceiro ou pelos pais, e considerada somente culpa da jovem, também pode gerar um comportamento agressivo da parte dos envolvidos, visando a sua punição. No caso dos pais e parceiros serem os agressores, esses podem também julgar estar exercendo sua autoridade através da agressão física. 


\subsubsection{Realização de acompanhamento pré-natal}

Doze estudos buscaram informações sobre a realização de acompanhamento pré natal pelas gestantes (Alegria et al., 1989; Chalem et al., 2007; Chemello et al., 2001; Costa et al., 1999; Faloppa et al., 1994; Gama et al., 2002; Persona et al., 2004; Pinto et al., 2005; Rigol \& Santo, 2001; Viana, 2005; Zaganelli, 2006; Zanchi et al., 1999). Dentre esses, apenas quatro verificaram os índices de comparecimento à assistência pré-natal (Chalem et al., 2007; Chemello et al., 2001; Rigol \& Santo, 2001; Zanchi et al., 1999), que variaram de $8,8 \%$ (Chalem et al., 2007) a 95,04\% (Zanchi et al., 1999). Essa diferença entre os percentuais ainda fica mais acentuada quando se discorre sobre as adolescentes na faixa etária de dezesseis anos ou menos, pois, de acordo com o estudo de Chalem et al. (2007),79,1\% das jovens referiram não ter acompanhamento. Esse dado é alarmante, visto a importância para a saúde tanto da mãe quanto do bebê de um acompanhamento médico desde o início da gravidez. Pode-se supor que o baixo índice de adolescentes grávidas que relataram realizar acompanhamento esteja relacionando a diversos fatores, variando conforme cada caso. Entre eles, podemos destacar o difícil acesso às unidades de saúde, a vergonha de assumir a gestação e até mesmo o desconhecimento dessa condição e/ou a sua negação.

No entanto, sete estudos referiram a procura do atendimento pré-natal ainda no primeiro trimestre da gestação (Alegria et al., 1989; Chemello et al., 2001; Costa et al., 1999; Faloppa et al., 1994; Pinto et al., 2005; Rigol \& Santo, 2001; Viana, 2005), o que se considera adequado. Diante disso, seria interessante questionar os fatores contextuais e individuais que estariam favorecendo um acesso mais precoce aos serviços de saúde por parte dessas gestantes investigadas. Infelizmente, informações para esse questionamento não foram encontradas nos estudos analisados. De qualquer modo, tais achados permitem compreender as razões para a utilização de dados de prontuários das instituições de saúde para caracterizar essa população.

\subsubsection{Estado civil}

Apenas oito estudos não continham informações sobre o estado civil das participantes (Alegria et al., 1989; Aquino et al., 2003; Chemello et al., 2001; Goldenberg et al., 2005; Lima et al., 2000; Madi et al., 2001; Silveira et al., 2004; Velasco, 1998). Dos 15 estudos restantes, três relataram uma maior incidência de adolescentes sem companheiros (Beretta et al., 1995; Carniel et al., 2006; Ponte Junior \& Ximenes Neto, 2004). Como contraponto, seis trabalhos referiram uma maior incidência de participantes casadas e/ou com companheiros (Chalem et al., 2007; Gama et al., 2002; Pinto et al., 2005; Rigol \& Santo, 2001; Viana, 2005; Zanchi et al., 1999).

O fato de haver jovens grávidas sem companheiros revela que, em muitos casos, o pai da criança pode abandonar a parceira por medo e insegurança, ou então, 
a gravidez pode ser decorrente de um relacionamento passageiro, não sendo impossivel que o pai da criança, inclusive, desconheça a gravidez. De qualquer modo, parece ser mais fácil para o homem se afastar da situação.

Com relação às jovens que permaneceram com seus parceiros, não se descarta que isso esteja ocorrendo por obrigação (social, familiar, religiosa), por um maior amadurecimento dos parceiros e das jovens, ou ainda, pela existência de um sentimento de amor e comprometimento entre eles. De qualquer forma, tal condição é positiva, pois o pai do bebê pode tornar-se uma importante fonte de apoio para as gestantes.

Apenas Faloppa et al. (1994) compararam o estado civil das gestantes quando engravidaram e a sua situação no momento de realização do estudo, constatando que $60, \%$ delas eram solteiras quando engravidaram, cinco se casaram durante a gestação e quatro se amasiaram. No caso, a gravidez pode ter impulsionado os casais que assim o desejavam (ou que sofreram as pressões antes referidas) a assumirem/oficializarem o seu relacionamento.

Quatro estudos (Costa et al., 1999; Sabroza, 2002; Simões et al., 2003; Zaganelli, 2006) relataram o estado civil de sua amostra, de acordo com as faixas etárias definidas. Os dados evidenciaram que, entre as participantes mais velhas (entre 17 a 19 anos), a incidência de união estável foi maior, quando comparadas às participantes mais novas, talvez justamente pela maior maturidade e desejo de estabelecer um relacionamento de maior comprometimento, seguindo o modelo adulto.

\section{Considerações Finais}

Através do presente estudo, que objetivou revisar a produção bibliográfica brasileira de 1987 a 2007 sobre o perfil sócio-demográfico de gestantes adolescentes atendidas em instituições públicas de saúde, pode-se verificar a falta de diversas informações dessa natureza nos estudos pesquisados, o que impede uma caracterização ampla do perfil sócio-demográfico dessa clientela. Dentre os achados, destaca-se que a faixa etária considerada para análise foi de 10 a 19 anos, sendo o número de gestações na adolescência mais elevado entre jovens de 17 anos e de nível socioeconômico baixo. Além disso, as gestantes adolescentes em geral não estudam e não exercem atividade remunerada. A gestação freqüentemente não é planejada, mas também não é evitada, em função do não uso de métodos contraceptivos. A menarca ocorre por volta dos 13 anos e a vida sexual inicia perto disso, entre 14 e 19 anos, logo ocorrendo uma gravidez. Contrariamente ao que se costuma pensar, a maioria das gestantes têm um parceiro estável, especialmente 
as mais velhas. Situações de risco psicossocial, como usar álcool e/ou drogas, sofrer abuso físico ou sexual e tentar praticar aborto, estão presentes na gestação entre as adolescentes brasileiras.

A fim de se obter um perfil sócio-demográfico mais completo dessa clientela, 336 sugere-se que estudos futuros possam incluir outras informações relevantes para tal fim, tais como dados do parceiro ou pai do bebê e dos genitores da adolescente. É importante que as instituições de saúde possam registrar essas informações do contexto sócio-econômico, afetivo e familiar, que auxiliariam na compreensão de alguns achados encontrados no presente estudo e também na elaboração de políticas públicas e programas de intervenção.

Desse modo, incentiva-se a melhor exploração dessa temática, tendo em vista a escassez de trabalhos científicos já realizados sobre o tema no âmbito brasileiro e a proporção ainda expressiva de gestações entre adolescentes nesse contexto. Mais especificamente, sugere-se a realização de estudos dessa natureza em instituições privadas de saúde, a fim de se comparar as características das clientelas atendidas nesses dois sistemas de saúde.

\section{Referências}

Aberastury, A. \& Knobel, M. (1981). Adolescência normal: Um enfoque psicanalítico. 10ed. (S. M. G. Ballve, Trad.). Porto Alegre: Artes Médicas. (Obra original publicada em 1970). Alegria, F. V. L., Schor, N. \& Siqueira, A. A. F. de (1989). Gravidez na adolescência: estudo comparativo. Revista de Saúde Pública, 23 (6), 473-477.

Aquino, E. M. L., Heilborn, M. L., Knauth, D., Bozon, M., Almeida, M. da C., Araújo, J. \& Menezes, G. (2003). Adolescência e reprodução no Brasil: A heterogeneidade dos perfis sociais. Cadernos de Saúde Pública, 19 (S2), S377- S88.

Beretta, M. I. R., Denari, F. E. \& Pedrazzani, J. C. (1995). Estudo sobre a incidência de partos na adolescência em um município do estado de São Paulo. Revista Latino- Americana de Enfermagem, 3 (2), 181-191.

Carniel, E. de F., Zanolli, M. de L., Almeida, C. A. A. de \& Morcillo, A. M. (2006). Características das mães adolescentes e de seus recém-nascidos e fatores de risco para a gravidez na adolescência em Campinas, SP, Brasil. Revista Brasileira de Saúde Materno-Infantil, 6 (4), 419-426.

Chalem, E., Mitsuhiro, S. S., Ferri, C. P., Barros, M. C. M., Guinsburg, R. \& Laranjeira, R. (2007). Gravidez na adolescência: Perfil sócio-demográfico e comportamental de uma população da periferia de São Paulo, Brasil. Cadernos de Saúde Pública, 23 (1), 177-186.

Chemello, C. S., Tanaka, A. C. d’A., Buzzetti, M. C. \& De Lorenzi, D. R. S. (2001). Estudo da incidência de gravidez entre adolescentes no Município de São Marcos - RS. Revista Cientifica da AMECS, 10 (1), 33-38. 
Costa, M. C. O., Queiroz, S. de S., Santos, C. A. T., Barboni, A. R., Oliveira Neto, A. F., Rocha, G. R., Rocha, C. C. \& Ferreira, M. H. (1999). Condições de gestação, parto e nascimento em adolescentes e adultas jovens: Santa Casa. Revista Adolescência Latino-Americana, 1 (4), 242-251.

Faloppa, C. C., Sato, D. K., Souza, E. do C. de, Valmórbida, G. A., Petrelli, G. S., Inoue, H. Y., Gonçalves, K. R., Abe, K., Firmino, K., Catanho, M. M. J., Oliveira, R. C., Santilli, S. M. A., Dessunti, E. M. \& Pauli, D. S. de (1994). Gravidez na adolescência: Estudos de 50 casos atendidos na Maternidade Municipal de Londrina - PR. Semina, 15, 30-35.

Gama, S. G. N. da, Szwarcwald, C. L. \& Leal, M. do C. (2002). Experiência de gravidez na adolescência, fatores associados e resultados perinatais entre puérperas de baixa renda. Cadernos de Saúde Pública, 18 (1), 153-161.

Godinho, R. A., Schelp, J. R. B., Parada, C. M. G. de L. \& Bertoncello, N. M. F. (2000). Adolescentes e grávidas: onde buscam apoio? Revista Latino-Americana de Enfermagem, 8 (2) 25-32.

Goldenberg, P., Figueiredo, M. do C. T. \& Silva, R. de S. E. (2005). Gravidez na adolescência, pré-natal e resultados perinatais em Montes Claros, Minas Gerais, Brasil. Cadernos Saúde Pública, 21 (4), 1077-1086.

Levandowski, D. C. (2005). A transição para a parentalidade e a relação de casal de adolescentes. Tese de Doutorado não publicada. Programa de Pós-Graduação em Psicologia do Desenvolvimento, Instituto de Psicologia, Universidade Federal do Rio Grande do Sul, Porto Alegre, Brasil.

Lima, M. S. de S., Kobata, C. M. \& Silvestrini, W. S. (2000). Perfil de adolescentes grávidas, internadas em dois hospitais públicos. A Folha Médica, 119 (4), 59-65.

Madi, J. M., Bertotto, M. S. \& Ribeiro, E. (2001). Aspectos familiares observados na gravidez da adolescente: Identificação de algumas características de importância epidemiológica. Revista Científica da AMECS, 10 (1), 1-7.

Papalia, D. E., Olds. S. W. \& Feldman, R. (2006). Desenvolvimento humano. (D. Bueno, Trad.). 8ed. Porto Alegre: Artes Médicas. (Obra original publicada em 2001)

Persona, L., Shimo, A. K. K. \& Tarallo, M. C. (2004). Perfil de adolescentes com repetição de gravidez atendidas num ambulatório pré-natal. Revista Latino-Americana de Enfermagem, 12 (5), 745-750.

Piccinini, C. A., Rapoport, A., Levandowski, D. C. \& Voigt, P. R. (2002). Apoio social percebido entre mães adolescentes e adultas. Psico, 33 (01) $09-35$.

Pinto, L. F., Malafaia, M. de F., Borges, J. A., Baccaro, A. \& Soranz, D. R. (2005). Perfil social das gestantes em unidades de saúde da família do município de Teresópolis. Ciência \& Saúde Coletiva, 10 (1), 205-213.

Ponte Junior, G. M. \& Ximenes Neto, F. R. G. (2004). Gravidez na adolescência no município de Santana do Acaraú - Ceará - Brasil: Uma análise das causas e riscos. Revista Eletrônica de Enfermagem, 6 (1), 25-37. Disponivel em http://www.fen.ufg.br, acesso em 21/09/2007.

Rigol, J. L. \& Santo, L. C. do E. (2001). Perfil das gestantes adolescentes atendidas em consulta de enfermagem. Revista Gaúcha de Enfermagem, 22 (2), 122-140.

Sabroza, A. R. (2002). Gravidez inoportuna: Retrato psicossocial de gestantes adolescentes no municipio do Rio de Janeiro (1999/2001). Dissertação de Mestrado não publicada. Escola Nacional de Saúde Pública, Fundação Oswaldo Cruz, Rio de Janeiro, Brasil. 
Sabroza, A. R., Leal, M. C., Gama, S. G. N. \& Costa, J. V. (2004). Perfil sociodemográfico e psicossocial de puérperas adolescentes do Município do Rio de Janeiro, Brasil -1999-2001. Cadernos de Saúde Pública, 20 (1), S112-120.

Silveira, I. P. da, Oliveira, M. I. V. \& Fernandes, A. F. C. (2004). Perfil obstétrico de adolescentes de uma maternidade pública no Ceará. Escola Anna Nery Revista de Enfermagem, $8(2), 205-210$.

Simões, V. M. F., Silva, A. A. M. da, Bettiol, H., Lamy-Filho, F., Tonial, S. R. \& Mochel, E. G. (2003). Características da gravidez na adolescência em São Luís, Maranhão. Revista Saúde Pública, 37 (5), 559-565.

WHO (World Health Organization). World health day safe motherhood [on-line]. Disponivel em http://www.who.int/docstore/world-health-day/en/pages1998/whd98 04.html, acesso em 03/11/2007.

Velasco, V. I. P. (1998). Estudo epidemiológico das gestantes adolescentes de Niterói. Dissertação de Mestrado não publicada. Escola Nacional de Saúde Pública, Fundação Oswaldo Cruz, Rio de Janeiro, Brasil.

Viana, G. G. A. (2005). Perfil epidemiológico das adolescentes grávidas atendidas na Unidade Básica de Saúde do Bairro Padre Palhano em Sobral - CE. Trabalho de Conclusão de Curso de Especialização e Residência em Saúde da Família, não publicado. Escola de Formação em Saúde da Família Visconde de Sabóia, Universidade Estadual do Vale do Acaraú, Sobral, Brasil.

Zaganelli, F. L. (2006). Aspectos do perfil social da gestação e do parto da adolescente e da mulher adulta e suas repercussões sobre o recém-nascido. Dissertação de Mestrado não publicada. Programa de Pós-Graduação em Ciências da Saúde, Faculdade de Medicina, Universidade Federal de Minas Gerais, Belo Horizonte, Brasil.

Zanchi, M. T., Carneiro, R. M. \& Menezes, R. E. (1999). Análise retrospectiva da gestação na adolescência no Hospital de Caxias do Sul. Revista Científica da AMECS, 8 (2), 11-15.

\section{Characterization of Socio-Demographic Profile of Pregnant Teenagers: Review of Brazilian Literature}

This study aims a bibliographic research of Brazilian studies published from 1987 to 2007, which scrutinizes the socio-demographic profile of pregnant teenagers attended in public health institutions. From a research done in search sites (Scielo, BVS-Psi, Google Schoolar), it was found 23 papers about this subject that were analyzed in relation to informed features of pregnant teenagers. In general the age average considered was from 10 to 19 years, and the numbers of pregnant girls was higher in low socioeconomic level in teenagers with 17 years old. It also became evident the lack of information about relational and familiar aspects, that can difficult the planning of interventions with this population. Furthermore, it is suggested that future studies could add other relevant information for the characterization of a broad socio-demographic profile, including information about adolescent's partner and parents.

KEYWORDS: Demographic Characteristics; Adolescent Pregnancy; Bibliographical Research. 